JIOM Nepal, Volume 41, Number 2, August 2019, page 8-10

\title{
Retrospective Review of Adrenalectomy in a Tertiary Center in Nepal
}

Pawan Dhital , Bipendra DK Rai, Manish Pradhan, Sujeet Poudyal, Suman Chapagain, Bhoj R Luitel, Pawan R Chalise, Uttam K Sharma, Prem R Gyawali

Department of Urology and Kidney Transplant Surgery, Maharajgunj Medical Campus, Tribhuvan University Teaching Hospital, Kathmandu, Nepal

\section{Corresponding author:}

Pawan Dhital, MBBS, MS

Department of Urology and Kidney Transplant Surgery, Maharajgunj Medical Campus, Tribhuvan University Teaching Hospital, Kathmandu, Nepal

Email: pawandhital21@gmail.com

Submitted : Oct 28, 2018

Accepted : Jun 7, 2019

\begin{abstract}
Introduction

Adrenal gland surgery is a challenging art as it encompasses diverse physiological derangements which are even life-threatening. Also, it remains a medical finesse to rightly identify the correct patient who will benefit from surgery which include strong biochemical and radiological resource. Soundly performed surgery will lead to oncologic and homeostatic correction leading to near normal course.
\end{abstract}

\section{Methods}

This is a retrospective analysis of adrenalectomy cohort over three years in a tertiary center in Nepal identifying diagnosis, perioperative management and postoperative course. The objective of this study is to review the presentation, histopathology and clinical outcomes of patients operated for adrenal mass in our center.

\section{Results}

There were 42 surgeries performed via open approach. The number of adenoma, phaeochromocytoma, carcinoma and non-functioning tumor were 13,16, 12 and one respectively. In most of the hypertensive patients, control was achieved with minimal drug and recurrences were observed in $25 \%$ of carcinoma in postoperative follow up.

\section{Conclusion}

A large proportion of operative adrenal mass are observed clinically in our setup. The surgical outcome is satisfactory but judicious perioperative management is essential.

Keywords: Adrenalectomy, adrenocortical carcinoma, phaeochromocytoma

\section{INTRODUCTION}

A drenal glands though seemingly small, possess vital role in homeostasis in human body. The hormones produced by this gland aldosterone, cortisol and catecholamines play important role in blood pressure, sodium, potassium and acid base balance. Adrenal was ignored in history of anatomy until Addison claimed Addisonian crises pertaining to adrenal in 1856 and it was then its significance was established.

The first planned adrenalectomy was performed only in 1914 by Perry Sargent. ${ }^{1}$ It was Gagner and colleagues who performed the first laparoscopic adrenalectomy in 1992.2,3 The basic indications of adrenalectomy include the functioning adrenal adenoma, carcinoma, phaeochromocytoma and adrenal incidentalomas when malignancy cannot be ruled out.
There is variation among the indication and technique of adrenalectomy in different centers as per expertize and logistics. Thus to study the presentation of the adrenal masses to our center and the indications and perioperative course of adrenalectomy done in our center we did a retrospective analysis of the same over the past three years.

\section{METHODS}

We performed a retrospective study including 42 patients who underwent adrenalectomy in Tribhuvan University Teaching Hospital, Kathmandu, Nepal over a duration of 40 months from February 2015 to June 2018.

The objective of our study was to assess clinical presentations, indications of adrenalectomy, final histopathological results, to delineate the short term complications and to review the follow up. 


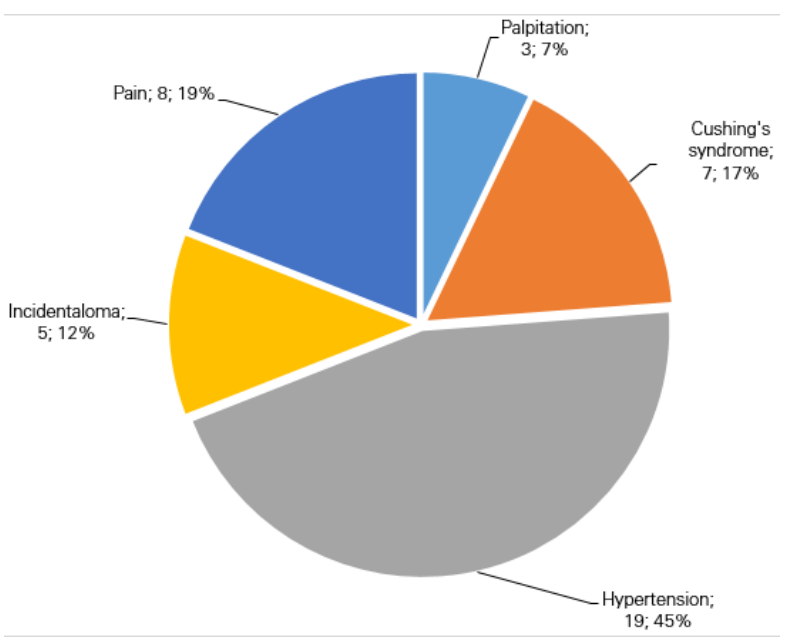

Figure 1. Clinical presentations

\section{RESULTS}

There were 22 males and 20 females. The most common presentation was hypertension (figure 1). The most common histopathological diagnosis was phaeochromocytoma (figure 2).

In 13 patients with adenoma, the size ranged from $1.5-5 \mathrm{~cm}$. Among 13 adenomas, cortisol, aldosterone secreting and non-functioning tumors were six, four and three respectively. After surgery, number of antihypertensive was reduced from four to one in three patients. There were no clinical or biochemical recurrences till end of one year.

In 16 cases with phaeochromocytoma, hypertension was the most common presentation. Diagnosis was made by imaging and functional marker 24 hour urinary vanelylmandellic acid and metanephrines. There were no familial, bilateral or multifocal lesion thus functional imaging were not done and genetic study was not available. Preoperative blood pressure optimization was done with Prazosin with sequential addition of metoprolol and amlodipine. Two liters of normal saline was infused overnight before surgery routinely. Open approach with minimal tumor handling was done and post-operative care done in ICU. There was no target organ compromise during

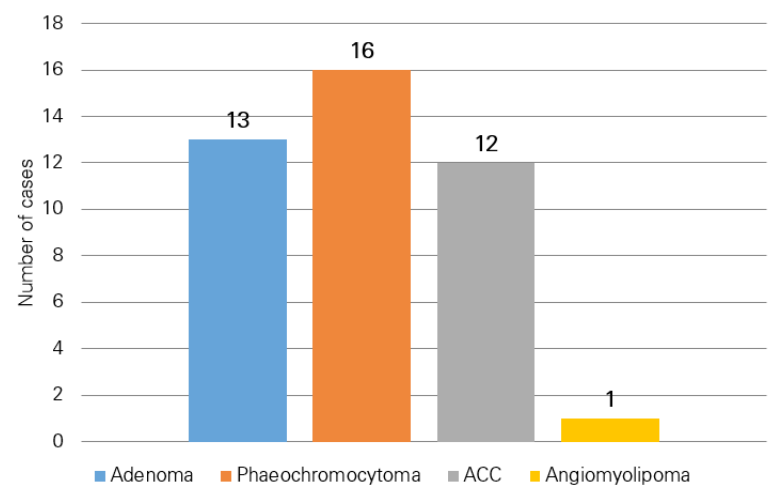

Figure 2. Histopathological diagnosis the perioperative period however most of the patients had grade I-II hypertensive retinopathy. Out of 16, 12 required ionotropes, nine required antihypertensives and five were normotensive in the immediate postoperative period.

All patients were followed within two weeks then yearly with metanephrines and CT abdomen at six months. No recurrence has been observed. Four patient were lost in follow up.

In 12 patients with adrenocortical cancer (ACC), age ranged from 35 to 49 years, pain and Cushing's syndrome were the predominant presentations. Provisional diagnosis was made from CT imaging in 10/12 except two cases of Cushing syndrome in which it was a retrospective diagnosis by histopathologic examination. The size ranged from $5-18 \mathrm{~cm}$. Complete excision was performed in all via open approach.

During follow up two recurrences and one mortality due to recurrence has been observed. Three patients were lost to follow up.

\section{DISCUSSION}

The preoperative evaluation includes thorough biochemical evaluation of the hypothalamicpituitary-adrenal axis. ${ }^{3}$ Electrolyte abnormalities and hypertension in patients with functioning adrenal mass should be corrected before surgery. CT abdomen with washout studies in adrenal protocol is of choice in making radiological diagnosis. Functional imaging in form of meta-iodobenzyl guanidine (MIBG) test and fluorodeoxyglucose positron emission tomography (FDG PET) helps to find multifocal, metastatic and bilateral tumors.

Adrenal-vein sampling (AVS) should be performed to confirm the laterality of secretion or excess secretion in case of more than 40 years age, bilateral no mass or multinodularity. ${ }^{4}$ Alternative imaging modality, ${ }^{11} \mathrm{C}$-metomidate PET, can also be used to identify the source of excess aldosterone secretion. Conn's syndrome preparation includes spironolactone or eplerenone. Potassium supplementation, blood pressure and heart rate optimization has to be done before surgery. ${ }^{5}$

Preoperative treatment of patients with hypercortisolaemia can include various medications but adrenal inhibiting drugs such as steroids, mitotane, metyrapone, ketoconazole and/or mifepristone are the drugs most likely to be used. Electrolytes, intravascular volume status and blood pressure should be carefully managed and optimized as much as possible. ${ }^{6}$ Characteristics of benign adrenal tumors include small size, smooth borders, lack of invasion of adjacent structures and no lymphadenopathy or evidence of metastatic disease. Additionally, necrosis, heterogeneity, calcification, lipid poor and poor washout are feature of malignancy. ${ }^{7}$ The risk of 
a primary $A C C$ in a tumor $<4 \mathrm{~cm}, 4-6 \mathrm{~cm}$ and $>6 \mathrm{~cm}$ are 2,6 and $25 \%$ respectively. ${ }^{8}$

The median size of benign aldosteronomas is $1.5 \mathrm{~cm}$ (range of 0.4-3.1 cm). The median size of aldosterone producing ACCs that only produce excess levels of is $6.5 \mathrm{~cm}$ (range of $3.9-18.0 \mathrm{~cm}$ ). ${ }^{9}$

Removal of a portion of the adrenal gland to enable the preservation of at least $30 \%$ of the structure for residual function has been described in patients with bilateral phaeochromocytoma and multiple endocrine neoplasia type 2 or Von Hippel-Lindau syndromes. ${ }^{10}$ A review of six studies including 203 patients who underwent bilateral adrenalectomy reported an incidence of addisonian crisis of $9 \% .{ }^{11}$

Data on surgical choice for ACC are limited and hampered by referral bias. The first International Adrenal Cancer Symposium in 2003 stated that there was no role for laparoscopic removal of a known or probable ACC. ${ }^{12}$ American Association of Endocrine Surgeons in 2009 advocate for open adrenalectomy as the procedure of choice. Conversely, the European Society of Endocrine Surgeons and the European Society for Medical Oncology suggest laparoscopic adrenalectomy for the adrenal tumors stage 1 or 2 less than $8-10 \mathrm{~cm}$ if an $\mathrm{R} 0$ resection is performed. ${ }^{13}$ Neither guideline addresses the differentiation of stage 1-2 ACCs from microscopic or unappreciated stage 3 ACC preoperatively, which occurs in $25 \%$ of patients.

Leboulleux and co-workers reported peritoneal carcinomatosis in $60 \%$ of patients with laparoscopic compared with $25 \%$ of patients undergoing open adrenalectomy. ${ }^{14}$ In contrast, two similarly designed studies from Italy reported comparable recurrencefree survival for laparoscopic adrenalectomy and open adrenalectomy in patients with stage 1 and 2 disease..$^{15}$ Thus, the existing literature is inconclusive regarding laparoscopic adrenalectomy versus open adrenalectomy for ACC. Until such time as conclusive data are available, an open approach is recommended for all adrenocortical tumors that cannot be classified as benign before surgery.

\section{CONCLUSION}

Adrenalectomy is a surgical challenge with respect to surgical anatomy and its diverse physiology and radiological feature which has significant impact on the accurate diagnosis, perioperative management and long term outcome. However, for malignant cases, open surgery is the standard of care.

\section{CONFLICT OF INTEREST}

None declared.

\section{REFERENCES}

1. Hergus S, Lynn J: Surgical Anatomy and Surgery of the Adrenal Gland. In Surgical Endocrinology. Edited by Lynn J, Bloom SR. London: Butterworth-Heinemann; 1993:458-467.

2. Greenblatt, R. B. in Search the Scriptures 3rd edn 45-48 (J. P. Lippincott, Philadelphia, 1977).

3. Zeiger, M. A. et al. The American Association of Clinical Endocrinologists and American Association of Endocrine Surgeons medical guidelines for the management of adrenal incidentalomas. Endocr. Pract. 15 (Suppl. 1), 1-20 (2009).

4. White, M. L. et al. The Role of Radiologic Studies in the Evaluation and Management of Primary Hyperaldosteronism. Surgery 144, 926-933 (2008)

5. Zarnegar, R. et al. The Aldosteronoma Resolution Score: Predicting Complete Resolution of Hypertension after Adrenalectomy for Aldosteronoma. Ann. Surg. 247, 511518 (2008).

6. Guerrieri, M. et al. Primary Adrenal Hypercortisolism: Minimally Invasive Surgical Treatment or Medical Therapy? A Retrospective Study with Longterm Followup Evaluation. Surg. Endosc. 24, 2542-2546 (2010).

7. Johnson, P. T., Horton, K. M. \& Fishman, E. K. Adrenal Mass Imaging with Multidetector CT: Pathologic Conditions, Pearls, and Pitfalls. Radiographics 29, 13331351 (2009).

8. [No authors listed] NIH State-of-the-science Statement on Management of the Clinically Inapparent Adrenal Mass ("incidentaloma"). NIH Consens. State Sci. Statements 19, 1-25 (2002).

9. Miller B,Doherty G. A-Surgical Management of Adrenocortical Tumours.Nature Reviews Endocrinology 10, 282-292.

10. Walz, M. K. Extent of Adrenalectomy for Adrenal Neoplasm: Cortical Sparing (Subtotal) versus Total adrenalectomy. Surg. Clin. North Am. 84, 743-753 (2004).

11. Ritzel, K. et al. Outcome of Bilateral Adrenalectomy in Cushing's Syndrome: A Systematic Review. J. Clin. Endocrinol. Metab. 98, 3939-3948 (2013).

12. Schteingart, D. E. Management of Patients with Adrenal Cancer: Recommendations of an International Consensus Conference. Endocr. Relat. Cancer 12, 667680 (2005).

13. Berruti, A. et al. Adrenal cancer: ESMO Clinical Practice Guidelines for Diagnosis, Treatment and Follow-up. Ann. Oncol. 23 (Suppl. 7), 131-138 (2012).

14. Leboulleux, S. et al. Adrenocortical Carcinoma: is the Surgical Approach a Risk Factor of Peritoneal Carcinomatosis? Eur. J. Endocrinol. 162, 1147-1153 (2010).

15. Porpiglia, F. et al. Retrospective Evaluation of the Outcome of Open Versus Laparoscopic Adrenalectomy for Stage I and II Adrenocortical Cancer. Eur. Urol. 57, 873-878 (2010). 